\title{
BMJ Open Knowledge, attitude and practices regarding health self-management among patients with osteogenesis imperfecta in China: an online cross- sectional survey
}

\author{
Yongjie Lai (D) , ${ }^{1}$ Wenchao Lu, ${ }^{2}$ Huijia Mao, ${ }^{3}$ Yueyang Zhang, ${ }^{4}$ Wai-kit Ming, ${ }^{5}$ \\ Yibo Wu (i) ${ }^{6,7}$
}

To cite: Lai Y, Lu W, Mao H, et al. Knowledge, attitude and practices regarding health selfmanagement among patients with osteogenesis imperfecta in China: an online crosssectional survey. BMJ Open 2021;11:e046286. doi:10.1136/ bmjopen-2020-046286

- Prepublication history and additional supplemental material for this paper are available online. To view these files, please visit the journal online (http://dx.doi.org/10.1136/ bmjopen-2020-046286)

YL and WL are joint first authors.

Received 26 October 2020 Accepted 12 August 2021

Check for updates

(C) Author(s) (or their employer(s)) 2021. Re-use permitted under CC BY-NC. No commercial re-use. See rights and permissions. Published by BMJ.

For numbered affiliations see end of article.

\section{Correspondence to} Dr. Wai-kit Ming; wkming@alumni.harvard.edu and

Yibo Wu;

bjmuwuyibo@outlook.com

\section{ABSTRACT}

Objectives Assessing the knowledge, attitude and practices (KAP) regarding health self-management among patients with osteogenesis imperfecta (OI) in China. Design An online cross-sectional survey.

Setting A structured questionnaire was distributed online through China-Dolls Center for Rare Disorders (CCRD), a non-governmental charity in China.

Participants Participants were all patients with 0 from CCRD. After stratified sampling according to the economic level of residential city, 901 patients were proportionally selected and 869 patients completed the survey (response rate: $96.4 \%$ ).

Main outcome measures Demographic characteristics and KAP information were gathered using a 5-point Likert scale. The score of each dimension of KAP was the sum of the scores of all included items. $X^{2}$ test or Fisher's exact test was used to compare scores of different items. Ordinal regression was employed to determine the significant factors influencing KAP. Results A total of 802 questionnaires were included for analysis. Male respondents accounted for $57.1 \%$, and $29.1 \%$ of respondents were of $26 \sim 30$ years. More than half $(50 \sim 60 \%)$ of patients knew the main symptoms of $0 \mathrm{l}$ and the treatment of Ol-related complications. Almost $80 \%$ of patients showed positive attitude. Nearly $60 \sim 70 \%$ of the patients were relatively able to take appropriate practice regarding 0 I management. There was a significant correlation between scores of knowledge and attitude, attitude and practice, as well as knowledge and practice. Patients aged 26 30 years, from urban areas and big cities, had higher KAP scores. Male patients showed better performance in knowledge, and highly educated patients perform better in practice.

Conclusions Patients with 0I did not have sufficient knowledge on disease care and up-to-date caring guidelines, and their practice regarding health selfmanagement also needs to be improved. Gender, age, educational level and economic level of residence can affect the level of KAP for patients, thus developing targeted and tailored programmes for patients with $0 \mathrm{l}$ is highly recommended.
Strengths and limitations of this study

- This is the first cross-sectional study to assess the knowledge, attitude and practices (KAP) regarding health self-management among patients with osteogenesis imperfecta (OI) in China.

- Our results suggest the importance of developing targeted educational programmes about health selfmanagement for patients with 0 I in China.

- The selection bias may exist in this study, despite the large sample size.

- Due to the limited length of the questionnaire, only few variables influencing KAP were included in this study.

\section{INTRODUCTION}

Osteogenesis imperfecta (OI), or 'brittle bone disease', is an inherited connective tissue disorder with a prevalence of about $1 / 15000 \sim 1 / 20000$ in newborns. ${ }^{1}$ In China, it is estimated that there are more than 100000 patients with $\mathrm{OI}^{2}{ }^{2}$ Individuals with OI have low bone mass and reduced bone material strength, which leads to increased bone fragility, repeated fractures and bone deformity. ${ }^{3}$ Although the primary clinical manifestation involves the skeleton, $\mathrm{OI}$ is a generalised connective tissue disorder, which is often accompanied by secondary features, including blue sclerae, conductive or sensory hearing loss, dentinogenesis imperfecta, pulmonary function impairment, cardiac valve abnormalities and muscle weakness. ${ }^{45}$ OI is most commonly caused by mutations in genes encoding the chains of type I collagen or proteins involved in post-translational modification of type I collagen. ${ }^{6}$

The main objectives of treatment in patients with OI are to decrease fracture incidence, relieve bone pain, promote mobility 
and growth, and ultimately improve the social participation and quality of life (QoL). ${ }^{78}$ This requires a multidisciplinary approach, using physiotherapy, rehabilitation, orthopaedic surgery, pharmacological interventions, and monitoring nutrition with calcium and vitamin D supplementation. ${ }^{9} 10$ Bisphosphonate is proven to be helpful to increase bone mass and to some extent reduce fracture risk. ${ }^{11}$ However, it seems less effective in certain types of OI, particularly for OI type 6 , and its response is worse in adult patients with OI than most paediatric cases. ${ }^{12} 13$ Other new pharmacological treatments are being developed, including denosumab, cathepsin $\mathrm{K}$ inhibition, growth hormone, teriparatide, and gene and cell therapy approaches. $^{14}$

The self-management of patients with OI for their health is very important, which helps them to effectively manage their illness and improve health outcomes. In order to prevent fractures, patients should adhere to medical treatment and be careful in daily activities. It is essential to continue regular follow-up care under the guidance of experienced doctors. ${ }^{8}$ Bone mass measurement needs to be performed regularly to predict their condition and clinical prognosis. ${ }^{815}$ Additionally, patients need to carry out appropriate rehabilitation training and protect their teeth and hearing. ${ }^{89}$ Patients' mental health is equally important, as facing the disease with optimistic attitudes helps patients to receive treatment actively. ${ }^{16}$ Overall, the level of self-management of patients and their families for their health plays an essential role in the treatment and prognosis of OI.

Lack of up-to-date knowledge, wrong attitudes and malpractice for OI management may play an important role in patients' non-compliance with treatment and failure to achieve therapeutic goals. Knowledge, attitude and practice (KAP) theory believes that enhancing the level of knowledge can change the attitude of individuals and then affect practice, which is the principle of this study. ${ }^{17} \mathrm{KAP}$ surveys are frequently used in health-seeking behaviour research, and they are very useful for assessing distribution of community knowledge in large scale and for evaluating changes in knowledge after education. ${ }^{18}$ To improve the outcome of the disease, it is very necessary to investigate the KAP level of health self-management for patients. To our knowledge, this is the first study to focus on this point. This study aimed to investigate the level of KAP regarding OI disease and its management for patients, identify the factors influencing the KAP level and come up with methods to improve their health selfmanagement level.

\section{METHODS}

\section{Study design}

This was a non-interventional, cross-sectional, stratified sampling online survey for patients with OI in China. The survey was conducted from 24 September to 30 September 2019, based on the online community built by
China-Dolls Center for Rare Disorders (CCRD), a public welfare organisation in China.

\section{Study population}

Participants in this study were all patients with OI registered and certified in CCRD. This organisation has built a network community of patients with $\mathrm{OI}$ in China, where we published information from 1 to 20 September 2019 to recruit patients to join in the survey. Patients who were willing to participate in the study needed to submit personal information, including medical history, city of residence and contact information, according to the requirements of the recruitment notice.

The inclusion criteria of the respondents were: (1) diagnosed as OI, regardless of gender and age; and (2) able to understand the content of the questionnaire. We believed that the patients who passed the patient identity authentication, joined the online community and submitted personal information in accordance with the notice of patient recruitment met the above inclusion criteria. The exclusion criteria were: accompanied with other serious diseases which might affect the KAP of patients with OI, such as serious heart, liver and kidney disease. The exclusion of patients was performed according to the medical history they submitted.

\section{Study instrument}

The original questionnaire was designed by researchers by reviewing previous literature. After pre-investigation in a small scale, the questionnaire was modified and improved to form the final version. The face validity of the questionnaire was evaluated by a sample of 15 patients from CCRD through discussing on unclear items. The content validity was assessed by 23 experts, including doctors from hospitals, professors from the school of public health or medicine, government staff from China Association for Science and Technology, and staff from CCRD. The Kaiser-Meyer-Olkin value of the adjusted questionnaire was 0.842; the approximate $X^{2}$ value of the Bartlett spherical test was 12434.691 ( $\mathrm{p}<0.001)$, which indicated the questionnaire could use factor analysis. Exploratory factor analysis was used to test the structure and validity of the questionnaire, the principal component analysis was used to extract common factors and the most orthogonal rotation of variance was used to reduce the dimension. Finally, $56.045 \%$ of the variance was explained by the three factors. The Cronbach's $\alpha$ coefficient (a measure of internal consistency) was 0.951 , and the coefficients of three dimensions of KAP were 0.892, 0.856 and 0.911, respectively. After revision, the questionnaire exhibited good reliability and validity.

The final version of the questionnaire can be seen in the online supplementary file, which consisted of four aspects: (1) demographic characteristics (5 questions, including gender, age, educational level, living in urban or rural areas, and residential city); (2) patients' knowledge of OI management, mainly investigating the patients' understanding of fracture, complications, commonly 
used drugs and rehabilitation training (10 questions); (3) patients' attitude regarding OI management, mainly evaluating the patients' attitude towards drugs, surgical treatment, rehabilitation training and daily management (7 questions); and (4) patients' practice of OI management, aiming to evaluate the implementation of the above health management behaviour of patients (10 questions). Each aspect of KAP contained several items, which adopted a Likert 5-level scoring method. The highest score for each item was 5 points, and the lowest was 1 point. The total score of each dimension of KAP was obtained by adding the scores of all included items. Higher scores meant higher KAP levels.

\section{Sample size and sampling}

In order to address potential sources of bias, patients who met the inclusion criteria $(n=5036)$ were divided by the economic level of their residential city into three groups: 1714 patients from first-tier cities $(34.0 \%), 1482$ from second-tier cities (29.4\%) and 1840 from other cities $(36.5 \%)$. In the pre-investigation, the mean total KAP scores of the first, second, and third group were 107 $(\mathrm{SD}=17), 102(\mathrm{SD}=18)$ and $98(\mathrm{SD}=20)$, respectively. We used the following equation to calculate the minimum sample size for this simple and stratified random sampling:

$$
\mathrm{n}=\left(\frac{\mathrm{Z}_{\alpha / 2} \mathrm{CV}}{\varepsilon}\right)^{2}
$$

The value of coefficient of variation (CV) was calculated by dividing the weighted $\mathrm{SD}$ by the weighted mean score of all the three groups $(\mathrm{CV}=0.18$, weighted $\mathrm{SD}=18.4$, weighted mean score $=102.2$ ). Assuming an $\alpha$ of $5 \%$ and $\varepsilon$ (relative error) of $2 \%$, the sample size calculations indicated a minimum of 311 patients were required to power the study.

Finally, a total of 901 patients were randomly and proportionally selected from all three groups (307 from first-tier cities, 265 from second-tier cities and 329 from other cities).

\section{Data collection and quality control}

The questionnaire was uploaded to the online platform of 'Wen Juan Wang' (http:/ /www.wenjuan.com/), generating a link to the questionnaire. The link was distributed to the included patients on 24 September 2019, valid for 7 days. Each Internet protocol (IP) address can complete the questionnaire only once, and only patients with $\mathrm{OI}$ in the group have access to the questionnaire. The submitted invalid questionnaires were excluded according to the following criteria: (1) the answer time is less than $120 \mathrm{~s}$; (2) the demographic information is incomplete or irrelevant to the survey; and (3) choosing the same option for 15 or more consecutive items in questions $6-8$.

\section{Statistical analysis}

The statistical analysis was completed using SPSS V.25.0 software. Frequency and percentage were used to describe the qualitative data, mean $\pm \mathrm{SD}$ was used to describe the quantitative data. The $\mathrm{X}^{2}$ test or Fisher's exact test was

\begin{tabular}{lc}
\hline $\begin{array}{l}\text { Table } 1 \\
(\mathrm{n}=802)\end{array}$ & Demographic characteristics of participants \\
\hline Characteristics & $\mathbf{n}(\%)$ \\
\hline Gender & $458(57.1)$ \\
\hline Male & $344(42.9)$ \\
\hline Female & \\
\hline Age & $82(10.2)$ \\
\hline Under 18 & $198(24.7)$ \\
\hline $18 \sim 25$ & $233(29.1)$ \\
\hline $26 \sim 30$ & $173(21.6)$ \\
\hline $31 \sim 40$ & $116(14.5)$ \\
\hline Over 40 & \\
\hline Education & $57(7.1)$ \\
\hline Elementary school & $170(21.2)$ \\
\hline Junior middle school & $301(37.5)$ \\
\hline Senior middle school & $274(34.2)$ \\
\hline Undergraduate or higher & \\
\hline Family residence & $530(66.1)$ \\
\hline Urban & $272(33.9)$ \\
\hline Rural & $273(34.0)$ \\
\hline City & $236(29.4)$ \\
\hline First-tier cities & $293(36.5)$ \\
\hline Second-tier cities & \\
\hline Other cities &
\end{tabular}

used to compare the composition ratio of scores between items in the questionnaire.

According to the score, the three dimensions of KAP were divided into three grades of 'good', 'medium' and 'poor'. For each dimension, the mean and SD of the total score are calculated, respectively. If the score of a certain dimension is less than or equal to the value of mean minus SD, we rated it as 'poor'; if the score was greater than that value and less than or equal to the value of mean plus SD, we rated it as 'medium'; if the score exceeds the value of mean plus SD, it would be rated as 'good'. The ordinal logistic regression models with an enter method were employed to examine the associations between different demographic characteristics and KAP score. The abovementioned five demographic variables were assigned scores, respectively, and were included in the analysis as independent variables. The dependent variable is the score grade of KAP, which assigned scores as follows: poor $=1$, medium $=2$ and $\operatorname{good}=3$. $\mathrm{P}<0.05$ (double tail) was statistically significant.

\section{Patient and public involvement}

Patients and/or the public were not involved in the design, conduct, reporting or dissemination plans of this research. 


\section{RESULTS}

\section{Demographic characteristics}

A total of 901 questionnaires were sent out and 869 questionnaires were retrieved, with a response rate of $96.4 \%$. Eight hundred two questionnaires were considered valid for analysis after exclusion. The demographic information is shown in table 1 . Of the 802 respondents, $57.1 \%$ were male and $29.1 \%$ were aged between 26 and 30 years old. A major proportion of the respondents had a high school degree or a higher academic degree (71.7\%). More than half $(66.1 \%)$ of the patients were from urban areas and more than one-third $(34.0 \%)$ were from firsttier cities.

\section{KAP about health self-management of 0 I}

Among the items regarding knowledge of OI management, the three items with lower average scores were item K6 (how to deal with the hearing loss associated with OI), item K7 (how to carry out oral care) and item K8 (the main adverse reaction of bisphosphonate). More than half $(56.1 \%)$ of the respondents knew that the disease might have other complications besides fracture. The $\mathrm{X}^{2}$ test results showed that the score composition ratio among item K4, item K6 and item K7 was significantly different $(\mathrm{p}<0.001)$, indicating that patients' knowledge of other complications was worse than fractures. Approximately $59 \%$ of the respondents knew how to judge and manage fractures, and the significance and process of bone mineral density measurement. But fewer patients were aware of the adverse reactions of bisphosphonate $(44.3 \%)$. Only $56.6 \%$ of patients knew some suitable rehabilitation approaches and their main functions (figure 1).
About $80 \%$ of patients showed a positive attitude towards health self-management, who recognised the importance of active surgery and drug treatment, professional rehabilitation, and appropriate exercise for disease control, and were confident to fight against the disease. About $82.9 \%$ of patients worried that their disease would deteriorate with age. Nearly $77.8 \%$ of patients were afraid of complications in OI, such as dental problems and hearing impairment (figure 2).

Regarding the practice of OI management, the two items with relatively low average scores were item P7 (observe the attention points in rehabilitation training) and item P9 (observe the dos and don'ts when using painkillers). Approximately $63 \%$ of patients were able to proactively learn the emergency management of fractures and can adhere to regular bone mineral density measurement. For dental care, $71.6 \%$ of patients persisted in brushing their teeth carefully and avoiding carbonated beverages, while the number of patients who could regularly go to the hospital to clean their teeth was relatively small $(65.8 \%)$. The proportion of patients who can adhere to regular rehabilitation training is close to $65.3 \%$, but relatively few patients can observe the attention points in rehabilitation training (57.1\%) (figure 3).

\section{Overall evaluation of KAP regarding health management of patients with 0 I}

In terms of scores in three dimensions of KAP, their Spearman correlation coefficients were 0.609 (knowledge-attitude, $\quad \mathrm{p}<0.01$ ), $0.919 \quad$ (knowledge-practice, $\mathrm{p}<0.01$ ) and 0.612 (attitude-practice, $\mathrm{p}<0.01$ ), which

$\mathrm{K} 1$. Osteogenesis Imperfecta is an autosomal dominant or recessive genetic disease.

K2. Patients with OI can predict whether the fetus is sick through prenatal diagnosis.

K3. In addition to easy fracture, the disease may also have other manifestations.

K4. How to judge and handle fractures.

K5. How to measure bone density and the importance of bone density measurement.

K6. How to cope with the hearing loss associated with the disease.

K7. How to carry out oral care for patients with the disease.

K8. Adverse reactions of bisphosphonate.

K9. What are the suitable rehabilitation methods for OI and their main function?

K10. How to alleviate the negative emotions caused by the disease.

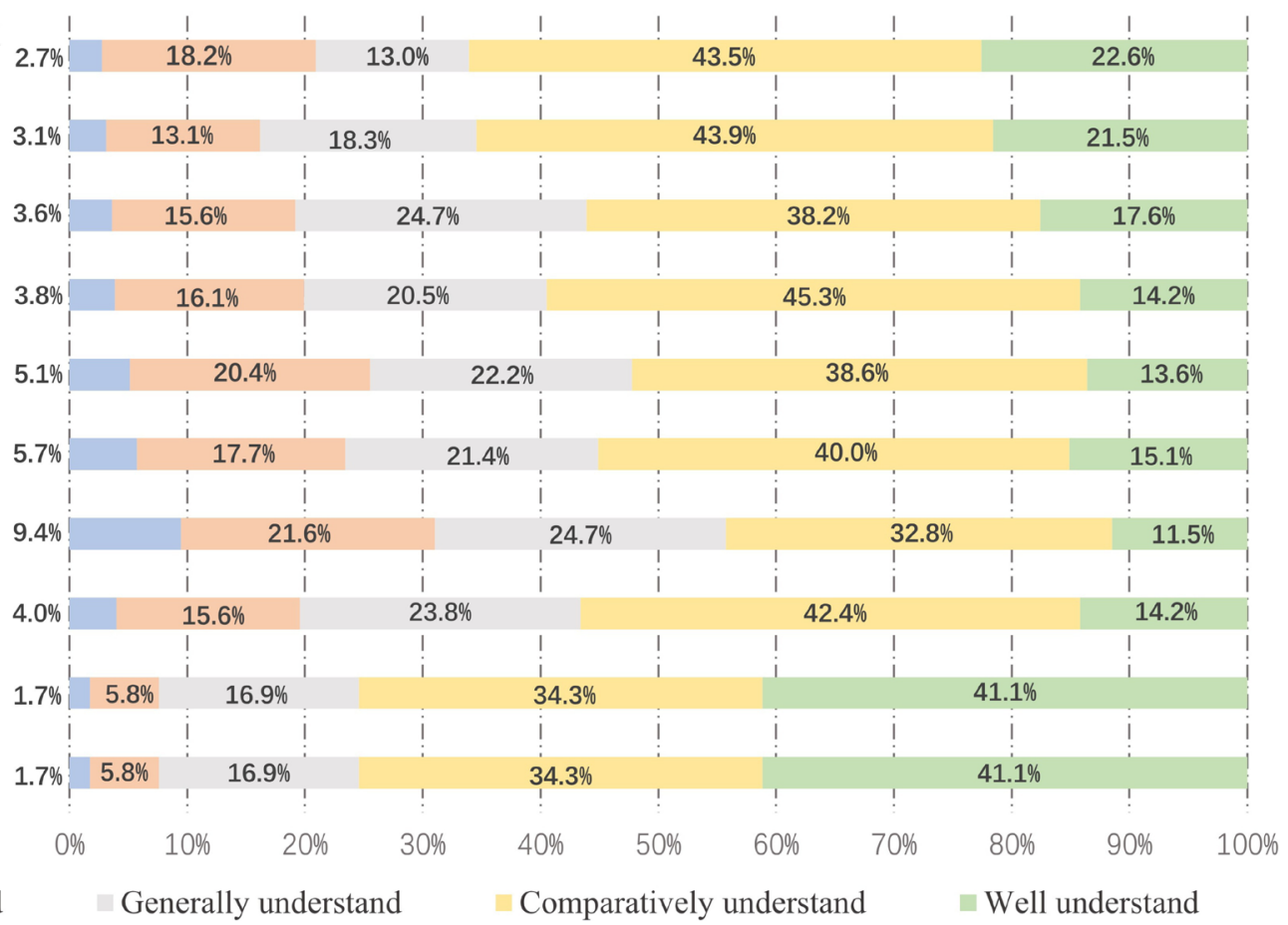

Do not Know Have heard

Figure 1 Response to statements regarding knowledge of health management of patients with osteogenesis imperfecta (OI). 
A1. Worried that the disease will deteriorate with age. 1.5\% $3.5 \% \quad 12.1 \%$

A2. Fear symptoms such as fractures, dental problems or hearing impairment

A3. Active surgery, drug therapy and professional rehabilitation are good for disease control.

A4. It is important to learn the precautions and adverse reactions of treatment drugs.

A5. Proper exercise is conducive to controlling the disease and improving quality of life.

A6. Regular follow-up visits are important for the treatment and you can insist on regular review.

A7. Have confidence to fight against the disease.

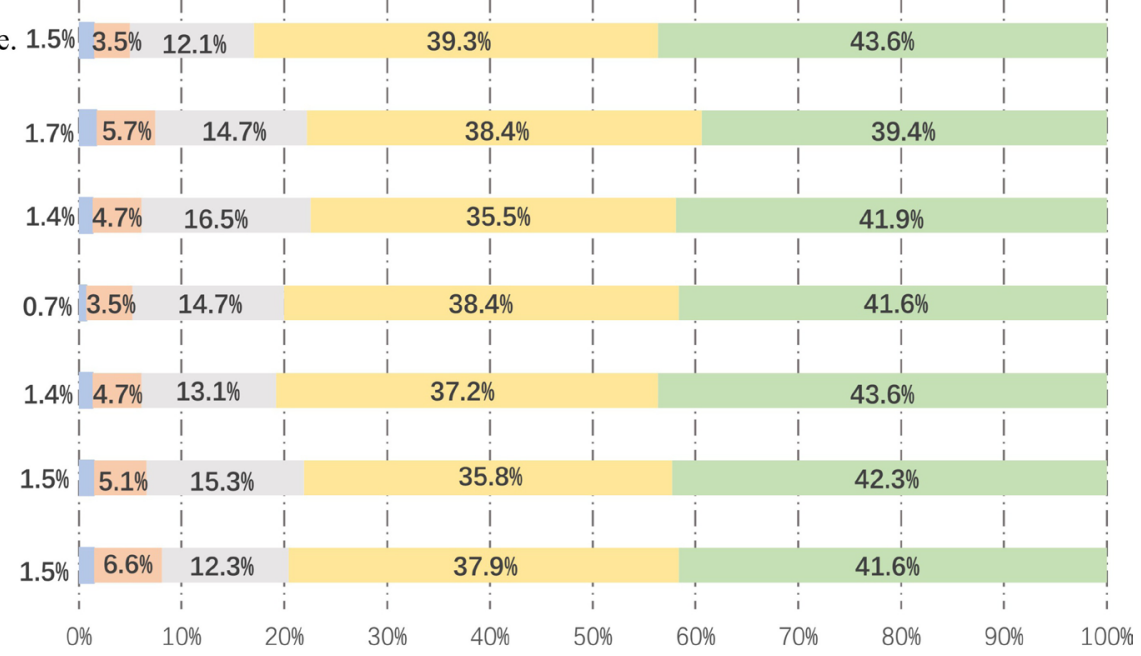

Strongly Disagree $\quad$ Comparatively disagree $\quad$ Neutral $\quad$ Comparatively Agree $\quad$ Strongly agree

Figure 2 Response to statements regarding attitude of health management of patients with osteogenesis imperfecta.

showed that the correlation between the three was significant. The mean \pm SD scores of KAP were $35.3 \pm 7.6$ (total

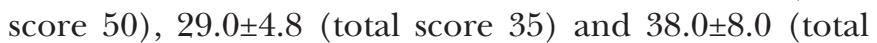
score 50). For the dimension of knowledge, the score less than or equal to 27.7 was rated as 'poor', greater than 27.7 and less than 42.9 was'medium', and greater than or equal to 42.9 was 'good'. The scores of the other two dimensions were rated using the same method based on the two values of mean $\pm \mathrm{SD}$. The distribution of KAP scores of patients with OI in different categories is shown in figure 4.

Table 2 shows the results of ordinal regression. It can be seen that gender, age, place of residence and educational level were important factors that affected the KAP level of patients. Female patients showed worse knowledge (OR 0.72, 95\% CI 0.53 to 0.98). Compared with patients over the age of 40 years, patients aged 26-30 years had a higher level of knowledge (OR 2.20, 95\% CI 1.36 to 3.55 ), better attitudes (OR 2.20, 95\% CI 1.38 to 3.50 ) and better practices (OR 2.07, 95\% CI 1.31 to 3.27 ); while patients under the age of 18 years performed worse in knowledge (OR 0.42, 95\% CI 0.23 to 0.78 ) and practice (OR $0.46,95 \%$ CI 0.26 to 0.84 ). Compared with patients in rural areas, patients from the urban area had similar phenomena. Compared with highly educated patients, patients from junior middle school had a worse performance in the practice of health management (OR 0.60 , 95\% CI 0.41 to $0.90)$.

P1. Keep learning the cutting-edge progress of the treatment method of OI.

P2. Actively learn emergency management of fractures.

P3. Perform bone mineral density measurement regularly.

P4. Brush teeth on time (at least twice a day) and never drink carbonated drinks.

P5. Go to the hospital regularly (preferably once every six months) for dental cleaning.

P6. Adhere to regular rehabilitation training.

P7. Observe the attention points in rehabilitation training.

P8. Stick to the follow-up protocol.

P9. Observe the dos and don'ts when using painkillers.

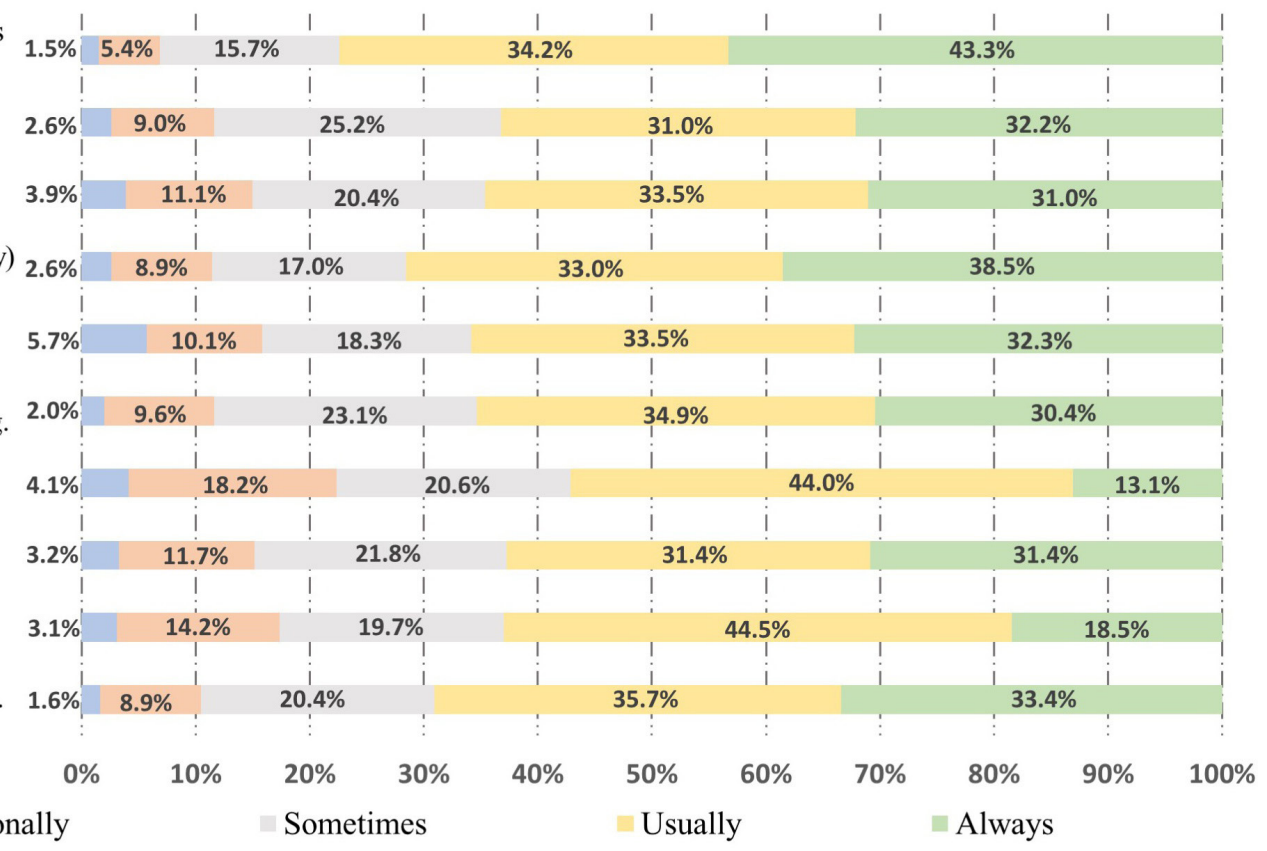

Hardly $\quad$ Occasionally $\quad$ Sometimes $\quad$ Usually
3 Response to statements regarding practice of health management of patients with osteogenesis imperfecta (OI). 


\section{Knowledge}

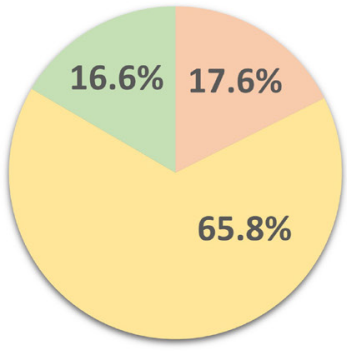

Attitude

$18.2 \% \quad 17.3 \%$

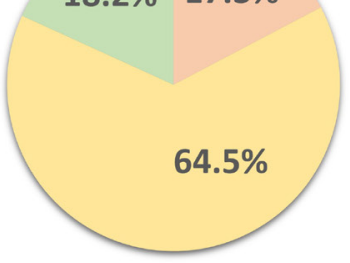

\section{Practice}

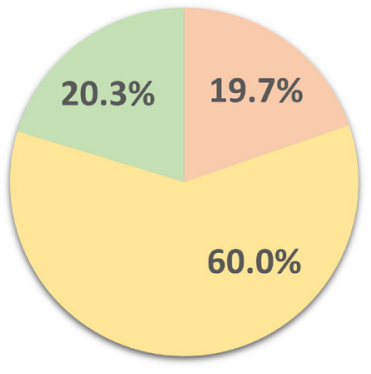

\section{Poor Medium Good}

Figure 4 The distribution of knowledge, attitude and practice scores of patients with osteogenesis imperfecta in different categories $(n=802)$.

\section{DISCUSSION}

This is the first KAP study regarding health management for patients with OI in China. Nearly $65 \%$ of the respondents knew the genetic characteristics of OI, and nearly $50 \% \sim 60 \%$ of patients knew the main symptoms of OI and the treatment of related complications. In terms of attitude, almost $80 \%$ of patients expressed varying degrees of concern about their condition, but they still had a positive attitude towards their disease management. A total of
$60 \% \sim 70 \%$ of the patients were able to carry out a variety of correct health self-management behaviours. Multivariate analysis showed that patients who were male, aged 26 30 years, with a college degree or above, living in cities and from first-tier cities were more likely to have a higher KAP level. The correlation among all three fields was found in the study, which implied the possibility of improving the health self-management level of patients with OI with educational interventions based on KAP theory.

Table 2 Multivariate analysis of KAP: results based on ordinal regression

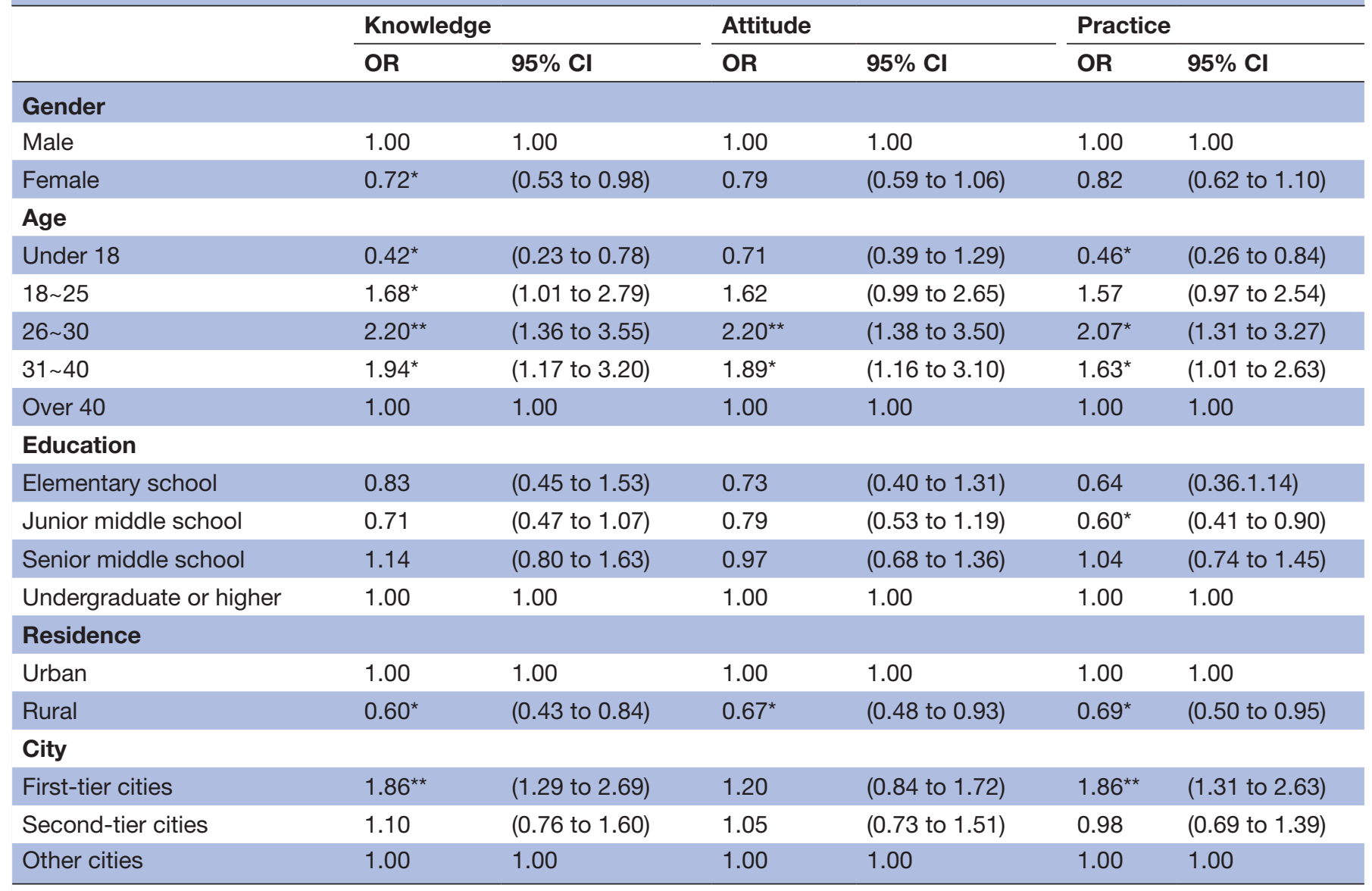

${ }^{*} \mathrm{P}<0.05 ;{ }^{* *} \mathrm{p}<0.01$. 
Additionally, given the significant correlation between knowledge and practice, educational programmes should be more practice oriented.

Most of the subjects still lacked knowledge related to OI management, which indicated the deficiency of health education for patients with OI in China. ${ }^{2}{ }^{19}$ The scattered distribution and inconvenience of patients to travel around make it difficult for the hospital to provide uniform guidance for patients. The relatively low educational level and poor internet skills greatly block their access to information. Meanwhile, the platform that connected nursing experts, doctors and patients' families has not yet been built, and there are few charitable organisations for patients with OI. Therefore, there is an urgent need to develop health education to meet the practical needs of patients in the internet era.

In our study, patients were much less acquainted with other OI complications than fractures, which might indicate that patients paid greater attention to fracture and bone density than other issues, or they might not even be aware of those problems. This phenomenon is also consistent with the results of the 2013 living condition survey of patients with OI conducted by CCRD. ${ }^{2}$ Fracture and fear of fracture are important factors affecting patients' QoL, ${ }^{16}{ }^{20}$ which run through the life and family of patients. ${ }^{21}$ However, paying too much attention to fracture might lead to neglect of the impact of other complications, which might pose potential risks to patients' prognosis or QoL. ${ }^{22}$ Therefore, the educational intervention should also emphasise knowledge regarding other common complications.

Most patients held a positive attitude towards the role of OI management, which will promote themselves to implement and adhere to correct behaviours of disease management in turn, according to KAP theory. However, about $80 \%$ of the patients were worried about their condition. This psychological condition includes the fear of fracture, the worry of reduced social support and isolation from others, which greatly affects patients' mental health and QoL. ${ }^{16}$ Worrying about the deterioration of disease can encourage patients to take practice appropriately, but continuous negative psychology is likely to have a negative effect on patients. It was previously investigated that $40.6 \%$ of the patients had suicidal thoughts, of which $3.8 \%$ often had suicidal thoughts, so the mental health of patients with OI deserves our attention. ${ }^{2}$ Various educational programmes are needed to improve patients' knowledge of disease symptoms and their ability of disease management, mitigating the negative impact of the disease on life. Furthermore, psychological counselling and social support are also essential for the improvement of psychological health and QoL of patients.

Patients' practice was not very good, and many reasons have contributed to this situation. First, the rehabilitation system of most public hospitals is not developed enough to meet patients' needs. ${ }^{19}$ Second, limited medical resources for OI force patients to travel far to other provinces or cities to treat their disease. Therefore, the risk of reinjury on the way, the cost of treatment, and the pressure of family care increase enormously and lead to many problems like medical insurance reimbursement. ${ }^{23}$ Moreover, terrible economic conditions due to illness and poor level of accessible facility renovation in patients' families ${ }^{1924}$ extremely restrict their daily activities and rehabilitation training. Hence, this prompts the need for an improvement on the existing social security system in China.

The undesirable situation of patients' practice can also be attributed to some psychological factors. Excessively worrying about fracture will do harms to patients' adequate rehabilitation and exercise. ${ }^{25}$ Besides, one of the main reasons that hinders patients' physical exercise is doctors' recommendations of avoiding too much exercise for safety reasons. ${ }^{26}$ Hence, health professionals should carefully consider the way of providing suggestions about physical exercise to patients, to ensure that patients and their family members understand how to exercise fully and safely with less fear.

According to the above and related researches, the health self-management of patients relies on the help of their family members, ${ }^{27}$ the social life of whom can also be affected by patients' disease. ${ }^{2125}$ Therefore, the economic and medical support for patients' families, as well as the health education and psychosocial support for patients' family members, are clearly warranted. ${ }^{28}$

Our study found that male patients had better knowledge regarding health self-management and KAP scores significantly varied by different age groups. Highly educated patients showed better practice of health selfmanagement, which may partly explain why higher educational level was associated with better QoL in patients with OI. ${ }^{29}$ Patients in different regions showed significantly different KAP scores owing to the fact that patients' needs for medical services of rare disease, education, jobs and barrier-free facilities were more easily met in some urban areas or more developed first-tier cities. ${ }^{219}$ Therefore, the above factors should be taken into account when tailoring health educational interventions.

Our study has some limitations. First, the respondents of our study are all patients from CCRD who can often receive health information about OI management. Hence, using this group of patients to represent the whole Chinese patient population is bound to introduce selection bias, despite the large sample size and stratified sampling method. Therefore, the actual level of KAP of Chinese patients should be worse than the results of this study. Further research should include more patients from underdeveloped areas. Moreover, due to the limited length of the questionnaire, only few influencing factors were suggested in this study. We suggest that more potential influencing factors be included in further studies, such as occupation, economic level, the type of OI, and the type and condition of complications, so as to find more effective ways to improve patients' ability to manage health and to achieve individualised and accurate health education. 


\section{CONCLUSION}

Patients with OI did not have sufficient knowledge on disease care and up-to-date caring guidelines, which indicated the deficiency of health education for patients with OI in China. Most patients held a positive attitude but their practice was not very good. The correlation among all three fields of KAP was found in the study, and this implied the possibility of improving the health self-management level of patients with OI by educational intervention based on KAP theory. The level of KAP was better among patients who were aged 26 30 years, from urban areas and from first-tier cities. Male patients showed better knowledge, while patients with higher education perform better in practice. Hence, developing targeted and tailored programmes for patients with OI is highly recommended.

\section{Author affiliations}

${ }^{1}$ School of Pharmaceutical Sciences, Shandong University, Jinan, Shandong, China

${ }^{2}$ Department of Pharmacy, Beijing Chao-Yang Hospital, Beijing, China

${ }^{3}$ School of Pharmaceutical Sciences, Jiangxi University of Traditional Chinese

Medicine, Nanchang, Jiangxi, China

${ }^{4}$ School of Public Health, Shandong University, Jinan, Shandong, China

${ }^{5}$ Department of Infectious Diseases and Public Health, Jockey Club College of Veterinary Medicine and Life Sciences, City University of Hong Kong, Hong Kong, People's Republic of China

${ }^{6}$ Key Research Base of Philosophy and Social Sciences in Shaanxi Province, Health Culture Research Center of Shaanxi, Xi'an, Shaanxi, China

${ }^{7}$ School of Public Health, Peking University, Beijing, China

Acknowledgements This study was supported by the China-Dolls Center for Rare Disorders. We thank all patients who participated in our study. We also thank Jiaqi Wang for English language editing of this manuscript.

Contributors WM and YW conceived and designed the study. WL contacted with organisation, designed the questionnaire and gave it out. YL came up with the statistical methodology. YL, HM and YZ collected and analysed the data. YL, YZ and HM wrote the paper. YL, WL, WM and YW reviewed and edited the manuscript. YW supervised and got funding for this progress. YL and WL contributed equally to this paper. All authors read and approved the manuscript. All authors approved the final version of the manuscript.

Funding This study was supported by China Association for Science and Technology Graduate Science Communication Ability Promotion Project (grant no. kxyjskpxm2019024 and kxyjskpxm2019055) and Qingdao Key Health Discipline Development Fund (grant 2020B047), which played an important role in the revision of the questionnaire, the recruitment of investigators and the collection of data.

Competing interests None declared.

Patient consent for publication Not required.

Ethics approval All the respondents signed the informed consent. The ethical committee of Health Culture Research Centre of Shaanxi approved this study in August 2019 (JKWH-2019-23).

Provenance and peer review Not commissioned; externally peer reviewed.

Data availability statement Data are available upon reasonable request.

Supplemental material This content has been supplied by the author(s). It has not been vetted by BMJ Publishing Group Limited (BMJ) and may not have been peer-reviewed. Any opinions or recommendations discussed are solely those of the author(s) and are not endorsed by BMJ. BMJ disclaims all liability and responsibility arising from any reliance placed on the content. Where the content includes any translated material, BMJ does not warrant the accuracy and reliability of the translations (including but not limited to local regulations, clinical guidelines, terminology, drug names and drug dosages), and is not responsible for any error and/or omissions arising from translation and adaptation or otherwise.

Open access This is an open access article distributed in accordance with the Creative Commons Attribution Non Commercial (CC BY-NC 4.0) license, which permits others to distribute, remix, adapt, build upon this work non-commercially, and license their derivative works on different terms, provided the original work is properly cited, appropriate credit is given, any changes made indicated, and the use is non-commercial. See: http://creativecommons.org/licenses/by-nc/4.0/.

\section{ORCID iDs}

Yongjie Lai http://orcid.org/0000-0002-2242-0917

Yibo Wu http://orcid.org/0000-0001-9607-313X

\section{REFERENCES}

1 Forlino A, Marini JC. Osteogenesis imperfecta. Lancet 2016;387:1657-71.

2 China-Dolls Center for Rare Disorders. A survey report on the survival status of patients with osteogenesis imperfecta in China, 2013. Available: http://chinadolls.org.cn/attachment/download?id=28 [Accessed 23 April 2020].

3 Marini JC, Forlino A, Bächinger HP, et al. Osteogenesis imperfecta. Nat Rev Dis Primers 2017;3:17052.

4 Tournis S, Dede AD. Osteogenesis imperfecta - A clinical update. Metabolism 2018;80:27-37.

5 Van Dijk FS, Sillence DO, DJAjomgPA S. Osteogenesis imperfecta: clinical diagnosis, nomenclature and severity assessment. Am J Med Genet A 2014;164A:1470-81.

6 Van Dijk FS, Pals G, Van Rijn RR, et al. Classification of osteogenesis imperfecta revisited. Eur J Med Genet 2010;53:1-5.

$7 \mathrm{Li} \mathrm{H}-\mathrm{J} \mathrm{HX}-\mathrm{Z}$, Du Q, et al. Health management in children with osteogenesis imperfecta. TMR Aging 2020;2:52-8.

8 Monti E, Mottes M, Fraschini P, et al. Current and emerging treatments for the management of osteogenesis imperfecta. Ther Clin Risk Manag 2010;6:367-81.

9 Marr C, Seasman A, Bishop N. Managing the patient with osteogenesis imperfecta: a multidisciplinary approach. J Multidiscip Healthc 2017;10:145-55.

10 Ralston SH, Gaston MS. Management of osteogenesis imperfecta. Front Endocrinol 2020;10:924.

11 Biggin A, Munns CF, CJCor M. Long-term bisphosphonate therapy in osteogenesis imperfecta. Curr Osteoporos Rep 2017;15:412-8.

12 Celik NB, Gonc N, Ozon A, et al. Treatment response to long term antiresorptive therapy in osteogenesis imperfecta type VI: does genotype matter? J Pediatr Endocrinol Metab 2020;33:1617-24.

13 Shi CG, Zhang Y, Yuan W, WJAjot Y. Efficacy of bisphosphonates on bone mineral density and fracture rate in patients with osteogenesis imperfecta: a systematic review and meta-analysis. Am J Ther 2016;23:e894-904.

14 Marom R, Lee Y-C, Grafe I, et al. Pharmacological and biological therapeutic strategies for osteogenesis imperfecta. Am J Med Genet C Semin Med Genet 2016;172:367-83.

15 Huang RP, Ambrose CG, Sullivan E, et al. Functional significance of bone density measurements in children with osteogenesis imperfecta. J Bone Joint Surg Am 2006;88:1324-30.

16 Tsimicalis A, Denis-Larocque G, Michalovic A, et al. The psychosocial experience of individuals living with osteogenesis imperfecta: a mixed-methods systematic review. Qual Life Res 2016;25:1877-96.

17 Demaio AR, Dugee O, Amgalan G, et al. Protocol for a national, mixed-methods knowledge, attitudes and practices survey on noncommunicable diseases. BMC Public Health 2011;11:961.

18 Cappon S, L'Ecluse C, Clays E, et al. Female genital mutilation: knowledge, attitude and practices of Flemish midwives. Midwifery 2015;31:e29-35.

19 Dong ZB. Research on osteoporosis imperfecta survival status, needs and security. [In Chinese. Shandong University, 2018.

20 Song Y, Zhao D, Li L, et al. Health-related quality of life in children with osteogenesis imperfecta: a large-sample study. Osteoporos Int 2019;30:461-8

21 Hill M, Lewis C, Riddington M, et al. Exploring the impact of osteogenesis imperfecta on families: a mixed-methods systematic review. Disabil Health J 2019;12:340-9.

22 Matsushita M, Mishima K, Yamashita S, et al. Impact of fracture characteristics and disease-specific complications on health-related quality of life in osteogenesis imperfecta. J Bone Miner Metab 2020;38:109-16.

23 Chinese Organization for Rare Disorders. Reports on drug access for rare disease, 2019. Available: http://www.raredisease.cn/source! downSource?id=8a9d475463869c1901692e1b43ac049e [Accessed 23 Apr 2020].

24 Huazhong University of Science and Technology.. An investigation report on rare diseases in China, 2018. Available: http://images.cdb. org.cn/editor/20180228/files/2018\%E7\%BD\%95\%E8\%A7\%81\% 
E7\%97\%85\%25\%20E8\%B0\%83\%E7\%A0\%94\%E6\%8A\%A5\% E5\%91\%8A.pdf [Accessed 23 April 2020].

25 Santos MCD, Pires AF, Soares K, et al. Family experience with osteogenesis imperfecta type 1: the most distressing situations. Disabil Rehabil 2018;40:2281-7.

26 Jones T, Theophile I, Price A. Barriers impacting on participation in physical activity for children with osteogenesis imperfecta: a study of child and parent perspectives. Cape Town, South Africa: Abstract from World Confederation of Physical Therapy Congress, 2017.
27 Dogba MJ, Dahan-Oliel N, Snider L, et al. Involving families with osteogenesis imperfecta in health service research: joint development of the OI/ECE questionnaire. PLoS One 2016;11:e0147654.

28 Dogba MJ, Bedos C, Durigova M, et al. The impact of severe osteogenesis imperfecta on the lives of young patients and their parents - a qualitative analysis. BMC Pediatr 2013;13:153.

29 Hald JD, Folkestad L, Harsløf T, et al. Health-related quality of life in adults with osteogenesis imperfecta. Calcif Tissue Int 2017:101:473-8. 